\title{
Psychiatric Co-morbidity in Patients With Irritable Bowel Syndrome at a Tertiary Care Center in Northern India
}

\author{
Yuman Kawoos, ${ }^{1 *}$ Zaid A Wani, ${ }^{1}$ Showkat A Kadla, ${ }^{2}$ Irfan A Shah, ${ }^{3}$ Arshad Hussain, ${ }^{1}$ M Maqbool Dar, ${ }^{1}$ Mushtaq A Margoob, ${ }^{1}$ and \\ Kouser Sideeq ${ }^{4}$ \\ Departments of ${ }^{1}$ Psychiatry, ${ }^{2}$ Gastroenterology, ${ }^{4}$ Preventive and Social Medicine, Government Medical College Srinagar, Srinagar, Jammu and \\ Kashimir, India; and ${ }^{3}$ Department of Neurology, Sheri-Kashmir Institute of Medical Sciences, Srinagar, Jammu and Kashmir, India
}

\section{Background/Aims}

Irritable bowel syndrome (IBS) is a chronic psycho-physiological disorder. It is considered to be the most common functional gastrointestinal disorder, and about 50-90\% of IBS patients have associated psychiatric co-morbidity. We aimed to study psychiatric co-morbidities in patients with IBS visiting a tertiary care center.

\section{Methods}

This was a cross-sectional case-control study conducted over a duration of one and a half years from January 2014 to July 2015. Patients were selected from the out-patient department of gastroenterology. About 160 patients with IBS who fulfilled the inclusion criteria and who gave written informed consent were selected as study cases. The healthy attendants of cases were selected as controls. A total of 200 controls were selected. Rome-III criteria were used to diagnose IBS. For diagnosing psychiatric disorders, we used the Mini International Neuropsychiatric Interview Schedule Plus.

\section{Results}

Mean age of our cases and controls was $39.7 \pm 11.4$ and $37.7 \pm 9.6$ years, respectively. Females outnumbered males in our cases as well as their controls by a ratio of 2:1 approximately. Psychiatric disorders were seen in $84.4 \%$ of IBS patients as compared to $41.5 \%$ in controls. Major psychiatric disorders seen in our patients were generalized anxiety disorders (30.0\%) and depression (28.0\%).

\section{Conclusions}

The majority of patients with IBS who present to a tertiary care center have co-morbid psychiatric disorders. We need to screen these patients for such co-morbidities and develop a holistic approach for better outcome in such cases.

\section{(J Neurogastroenterol Motil 2017;23:555-560)}

\section{Key Words}

Anxiety disorder; Co-morbidity; Depression; Gastrointestinal diseases; Irritable bowel syndrome

\section{Received: October 13, 2016 Revised: March 21, 2017 Accepted: April 2, 2017}

() This is an Open Access article distributed under the terms of the Creative Commons Attribution Non-Commercial License (http://creativecommons. org/licenses/by-nc/4.0) which permits unrestricted non-commercial use, distribution, and reproduction in any medium, provided the original work is properly cited.

*Correspondence: Yuman Kawoos, MD Department of Psychiatry, Government Psychiatric Diseases Hospital Srinagar, G1, Residential block, Srinagar, Jammu and Kashmir 190003, India Tel: +91-9419405478, Fax: +91-9419009329, E-mail: yumankawoos@ymail.com 


\section{Introduction}

Irritable Bowel Syndrome (IBS) is a chronic gastrointestinal (GI) disorder which is distinguished by repeated and inexplicable symptoms like diffuse or localized abdominal pain, constipation, diarrhea and urgency. ${ }^{1}$ It is thought to be one of the most common GI disorders, having an approximate prevalence of $1.1-29.2 \%$ in the community. ${ }^{2-5}$ The disorder amounts to a major burden on healthcare, and constitutes nearly half of the referrals to gastroenterology clinics. ${ }^{6-8}$

The mechanism of development of IBS has not been fully explained. Post-infectious inflammation, impaired gut motility, psychological distress; adverse life events and alteration in the brain-gut axis are among the various suggested pathophysiological explanations which may result in the development of this syndrome..$^{9-11}$ The treatment of this symptom complex consists of psychotherapy alone or a combination of psychotherapy with psychotropic drugs and symptomatic treatment for pain, constipation, and loose stools. Among the psychotherapeutic interventions, cognitive behavioral therapy is most effective for treatment of IBS. ${ }^{12}$

About $50-90 \%$ of IBS patients also have associated psychiatric ailments; most commonly anxiety disorders and depression. ${ }^{13} \mathrm{~Pa}-$ tients with this functional GI disorder have impairment in the health related quality of life. Also, patients with severe disease have higher prevalence of quality of life impairment. ${ }^{14}$ Studies have revealed that patients seeking medical consultation have a higher number and severity of symptoms ${ }^{15}$ and are more likely to be depressed and anxious. $^{16}$

The majority of the literature on IBS and associated psychiatric diseases comes from western studies. Very little research has been done on these interrelated disorders in this part of the world. Since major socio-cultural differences exist in the manifestation of these psychosomatic disorders, generalizing the outcomes of research from western studies will be unrevealing. Only few studies have been done in this area so far in India. Our aim therefore, was to strengthen the limited knowledge regarding the relation of psychiatric disorders with IBS in our region.

\section{Materials and Methods}

The present study was conducted in the Institute of Mental Health and Neurosciences in collaboration with the Department of Gastroenterology, Government Medical College Srinagar. It was a cross-sectional case-control study conducted for a length of about one and a half years from January 2014 to July 2015. Subjects for the study were selected from outpatients visiting the Gastroenterology department. After complete history and physical examination, subjects who fulfilled the inclusion criteria were selected as cases. The healthy attendants of cases were selected as controls. A total of 160 patients were selected as cases and 200 attendants served as controls. Organic causes were ruled out by the gastroenterologist after appropriate investigations wherever necessary. All of the cases were interviewed for socio-demographic parameters like gender, age, employment and educational level, marital status, and socioeconomic level. The socio-economic class was established by means of the Kuppuswamy's scale. The scale is used to ascertain the socio-economic level of urban populations, and takes into account education, occupation of the head of the family, and total income of the family. ${ }^{17}$ Education and occupation of the head of family did not change with time, but income has to be updated from time to time. ${ }^{18}$ All patients provided a written informed consent for inclusion in the research. The study was approved by the institute ethical committee of Government Medical College, Srinagar (IEC/GMCS Protocol No. 21/2012).

Subjects aged 18 years or above who satisfied the diagnostic criteria for IBS (Rome III criteria) and had no organic pathology on thorough investigations (upper GI endoscopy, colonoscopy, and abdominal ultrasonography) were included in our study as cases. Healthy attendants accompanying the patients (most of whom were first or second degree relatives of cases) were included to serve as controls. It was a sort of convenience sampling as the controls were interviewed in the same premises for presence of any psychiatric disorder. They were matched in terms of age and gender with cases. Patients diagnosed with any psychiatric disorder were referred to the psychiatric out-patient department for treatment.

Cases were diagnosed using Rome III criteria for IBS. ${ }^{19}$ For the diagnosis of psychiatric co-morbidity, we used the Mini International Neuropsychiatric Interview Schedule Plus. ${ }^{20,21}$

\section{Statistical Methods}

Data were entered into excel sheet designed by Microsoft Corporation, Redmond, WA, USA. Continuous variables were analyzed in the form of mean and standard deviation. Categorical variables were summed up as frequency and percentages. Chisquare test was applied to analyze the relationship between categorical variables. Fisher's exact test was used wherever Chi-square test did not meet the Cochrane criteria. Difference between 2 means was analyzed using the unpaired $t$ test. Odds ratio with $95 \%$ confidence interval was calculated to estimate the odds ratio for general- 
ized anxiety disorder (GAD) and major depressive episode (MDE). Open Epi (www.openepi.com/Menu/OE_Menu.htm) and Primer of Biostatistics were used for statistical analysis.

\section{Results}

The mean age of our IBS patients was $39.7 \pm 11.4$ years and that of their healthy controls was $37.7 \pm 9.6$ years. Females outnumbered males in our patients as well as their controls by a ratio of 2:1 approximately. An equal number of our patients and their controls belonged to rural or urban backgrounds. The maximum number of patients fitted into the upper-middle socio-economic class (Table 1).

In our study, psychiatric disorders were seen in 135 (84.4\%) of IBS patients as compared to $83(41.5 \%)$ of controls. Among the cases, $86.3 \%$ males and $83.6 \%$ females had a psychiatric co-mor-

Table 1. Socio-demographic Characteristics of Our Cases and Controls

\begin{tabular}{lccc}
\hline $\begin{array}{c}\text { Socio-demographic } \\
\text { characteristics }\end{array}$ & IBS cases & $\begin{array}{c}\text { Healthy } \\
\text { controls }\end{array}$ & $P$-value \\
\hline Mean age (yr) & $39.7 \pm 11.4$ & $37.7 \pm 9.6$ & 0.720 \\
Male:Female (ratio) & $44: 116(1: 2.6)$ & $74: 126(1: 1.7)$ & 0.056 \\
Residence (rural:urban) & $78: 82$ & $101: 99$ & 0.741 \\
Socio-economic status ${ }^{2}$ & & & \\
I & $3(1.9 \%)$ & $5(2.5 \%)$ & 0.850 \\
II & $72(45.0 \%)$ & $81(40.5 \%)$ & \\
III & $53(33.1 \%)$ & $77(38.5 \%)$ & \\
IV & $32(20.0 \%)$ & $37(18.5 \%)$ & \\
V & $0(0.0 \%)$ & $0(0.0 \%)$ & \\
\hline
\end{tabular}

${ }^{a}$ Kuppuswamy's scale.

IBS, irritable bowel syndrome.

$P$-value was insignificant for all socio-demographic variables. bidity while among controls $25.0 \%$ males and $50.0 \%$ females had co-morbid psychiatric illness. Major psychiatric disorders seen in our patients were GAD and MDE. GAD was seen in $30.0 \%$ of patients having IBS while MDE was present in $28.0 \%$. Our controls also had GAD and MDE as the major psychiatric disorder. GAD was present in $13.0 \%$ and MDE in $16.0 \%$ of our controls. Mixed anxiety depression was the next common psychiatric disorder seen in about $10.0 \%$ of IBS patients while only $2.0 \%$ of controls had the same disorder. Other common diagnoses seen in our patients were post-traumatic stress disorder, somatization disorder, obsessivecompulsive disorder, panic disorder, and adjustment disorder (Table 2).

The majority of our IBS patients had a mixed form of the illness; IBS-M, 68 patients $(42.5 \%)$. The least common form was the unclassified form of IBS; IBS-U, 16 patients (10.0\%). Other forms of IBS seen were constipation predominant IBS (IBS-C) in 40 patients $(25.0 \%)$ and diarrhea predominant IBS (IBS-D) in 36 patients $(22.5 \%)$. The relation between psychiatric disorders and various sub-types of IBS was not statistically significant as has been studied in some other studies on IBS (Table 3).

Table 2. Psychiatric Co-morbidity in Cases and Controls

\begin{tabular}{lccc}
\hline \multicolumn{1}{c}{ Psychiatric disorder } & IBS cases & Controls & $P$-value \\
\hline MDE & $44(27.5 \%)$ & $32(16.0 \%)$ & 0.011 \\
GAD & $48(30.0 \%)$ & $26(13.0 \%)$ & 0.000 \\
Mixed anxiety depression & $17(10.6 \%)$ & $4(2.0 \%)$ & 0.000 \\
Somatization & $15(9.4 \%)$ & $8(4.0 \%)$ & 0.063 \\
OCD & $1(0.6 \%)$ & $8(4.0 \%)$ & 0.047 \\
PTSD & $2(1.25 \%)$ & $0(0.0 \%)$ & 0.196 \\
Others & $8(5.0 \%)$ & $5(2.5 \%)$ & 0.037 \\
\hline
\end{tabular}

MDE, major depressive episode; GAD, generalized anxiety disorder; OCD, obsessive compulsive disorder; PTSD, post-traumatic stress disorder; Others, panic disorder, adjustment disorder, and dysthymia.

Table 3. Relation Between Psychiatric Disorders and Various Forms of Irritable Bowel Syndrome

\begin{tabular}{lccccc}
\hline & IBS-D & IBS-C & IBS-M & IBS-U & $P$-value \\
\hline MDE & 10 & 12 & 19 & 3 & 7 \\
GAD & 13 & 13 & 15 & 10.531 \\
Mixed anxiety depression & 4 & 2 & 3 & 1 & 0.236 \\
Somatisation & 4 & 0 & 1 & 0 & 0.414 \\
OCD & 0 & 0 & 6 & 1 & 0.287 \\
PTSD & 0 & 2 & 13 & 0 & 0.714 \\
Others & 0 & 5 & 1 & 0.770 \\
No disorder & 6 & 0 & 0.237 \\
\hline
\end{tabular}

IBS, irritable bowel syndrome; IBS-D, diarrhea predominant IBS; IBS-C, constipation predominant IBS; IBS-M, mixed IBS; IBS-U, unclassified IBS; MDE, major depressive episode; GAD, generalized anxiety disorder; OCD, obsessive compulsive disorder; PTSD, post-traumatic stress disorder; Others, panic disorder, adjustment disorder, and dysthymia.

$P$-value was not significant for any psychiatric disorder. Applying Fishers exact test, no difference was seen between different forms of irritable bowel syndrome for presence of psychiatric disorder 


\section{Discussion}

IBS is often considered to be the prototype of functional gastrointestinal disorders with about $50-90 \%$ of IBS patients having psychiatric co-morbidity. ${ }^{22}$ Patients showing manifestations of this disorder who report to a physician have a higher percentage of comorbid psychological disturbances than patients who do not look for medical help. ${ }^{15}$ The chronic and recurrent character of this disease may predispose patients to additional psychiatric disorders. The relation with psychiatric ailments may adversely affect the prognosis of this GI disorder leading to more frequent medical consultations by patients and escalating the health care costs. ${ }^{23}$

Females outnumbered males in our study by a ratio of almost 2:1 which is quite similar to the results seen in other studies, thereby showing that the disorder predominantly affects women. ${ }^{24,25}$ Researchers have hypothesized that sex hormones may affect the mechanisms that regulate the brain-gut-microbiota axis which is finally involved in the development of IBS. ${ }^{26}$ IBS is well thought to have a shared etiopathogenesis with other functional and somatic symptom disorders. ${ }^{27}$ It has been seen that females usually manifest psychiatric disorders as somatic complaints. In a study done in 2004 by Haug et al, ${ }^{28}$ it was found that female patients were having higher number of somatic symptoms than their male counterparts. Also, the study showed a strong relation of functional somatic symptoms with anxiety and depression.

The most frequent sub-type of IBS in our study was IBS-M followed by IBS-C, and IBS-D. IBS-U was the least common form seen in our study. These findings are in accordance with the results of some European and North American studies ${ }^{29,30}$ while they differ from several other studies. ${ }^{31,32}$ It has been seen that the type of IBS is largely determined by the population under study and varies among different regions. ${ }^{33}$

In our study, psychiatric disorders were seen in about $84.4 \%$ of IBS patients. This is quite high compared to the prevalence seen in western studies where the percentage of psychiatric disorders ranges from $40.0-60.0 \%,{ }^{34,35}$ but is comparable to the rates seen in Indian studies. ${ }^{36}$ In the study done by Prashant Singh et $\mathrm{al}^{36}$ in 2012, comorbid psychiatric disorders were found in $79.9 \%$ cases of IBS, which was considerably higher compared to $34.3 \%$ seen in controls. The higher prevalence of psychiatric disorders in our controls in comparison to the above study could be due to the fact that Kashmir being a conflict zone, the prevalence of stress and psychiatric diseases including anxiety disorders and depression is high when compared with other areas which are non-conflict zones. ${ }^{37,38}$
Another reason could be that people from developing countries report more somatic symptoms in comparison with developed countries, and IBS is one of the somatic symptom disorders. ${ }^{39}$ Also, the cases selected in our study were selected from a tertiary care center where more severe forms of the illness are seen, and it has been studied that the severity of functional GI disorders increases the likelihood of having co-morbid psychiatric disorders. ${ }^{14}$ In our cases, an almost equal number of males and females had psychiatric comorbidity, while in controls more females had a psychiatric illness as compared to males. This may be due to the higher prevalence of anxiety disorders and depression in females in the general population. ${ }^{40}$

The major psychiatric disorders seen in our cases were MDE and GAD which has been found in many other studies done on IBS. In a study done by Mayer et $\mathrm{al}^{41} 32.0 \%$ of patients of IBS presented with GAD symptoms. In another epidemiological study done in Hong Kong, the prevalence of GAD was seen to be considerably higher in subjects reporting symptoms of IBS compared to those who did not, and IBS was found to be associated with a 6-fold higher chance of having GAD.$^{42}$ In a study done by Kabra and $\mathrm{Nadkarni}^{43}$ in India, the prevalence of depression in IBS patients was about $37 \%$ which is slightly higher than our study. The probable reason being different scales were used to assess depression in the 2 studies. GAD was found in $31.0 \%$ of IBS patients in the same study which is quite similar to that of our study. ${ }^{43}$

The majority of patients had IBS-M with depression and anxiety as the major psychiatric co-morbidities, but no major differences were seen between the various types of IBS. In a study done in 2016 on IBS patients, it was seen that among the various types of IBS, anxiety was predominantly seen in the IBS-M sub-type but the sub-types did not differ significantly in depressive features. The higher rates of anxiety seen in IBS-M as compared to our study could be due to different scales used for assessing psychiatric comorbidities. $^{44}$

Keeping in view the high rates of psychiatric co-morbidity in patients of IBS as compared to the general population, all patients of IBS should be scrutinized for associated psychiatric disorders in order to build a holistic approach for managing these patients. By doing so, we will be able to manage these patients as a whole, rather than treating their individual symptoms which will improve the quality of life of patients having IBS.

Our study had a few limitations. We selected cases from a tertiary care specialty center where severe forms of illness are referred; therefore there is a higher chance of associated psychiatric co-morbidities in these patients compared to the ones in the general popu- 
lation or, those presenting to the primary care physicians. Also, most of our controls were relatives of patients, thus sharing the genetics which could have an influence on the prevalence of psychiatric comorbidity in the control group. Another limitation of our study is that we did not study the dietary habits of our cases which can have an influence on presentation of symptoms.

In conclusion, the majority of patients with IBS which present to a tertiary care center have co-morbid psychiatric disorders. Only a few of them receive specific psychological treatment. We emphasize screening of all patients with IBS for the presence of comorbidities and timely referral to a psychiatrist for appropriate treatment.

\section{Financial support: None.}

\section{Conflicts of interest: None.}

Author contributions: Yuman Kawoos and Zaid A Wani planned and conducted the study; Showkat A Kadla helped in collecting the data and diagnosing functional gastrointestinal disorders; Arshad Hussain, M Maqbool Dar, and Mushtaq A Margoob helped in interpreting the data and revising the manuscript; Irfan A Shah helped in drafting the manuscript; and Kouser Sideeq did the statistical analysis.

\section{References}

1. Corney RH, Stanton R. Physical symptom severity, psychological and social dysfunction in a series of outpatients with irritable bowel syndrome. J Psychosomatic Res 1990;34:483-491.

2. Talley NJ, Zinsmeister AR, Van Dyke C, Melton LJ. Epidemiology of colonic symptoms and the irritable bowel syndrome. Gastroenterology 1991;101:927-934.

3. Jones R, Lydyard S. Irritable bowel syndrome in the general population. BMJ 1992;304:87-90.

4. Saito YA, Locke GR, Talley NJ, Zinsmeister AR, Fett SL, Melton LJ 3rd. A comparison of the Rome and Manning criteria for case identification in epidemiological investigations of irritable bowel syndrome. Am J Gastroenterol 2000;95:2816-2824.

5. Oshima T, Miwa H. Epidemiology of functional gastrointestinal disorders in Japan and in the world. J Neurogastroenterol Motil 2015;21:320329.

6. Harvey RF, Salih SY, Read AE. Organic and functional disorders in 2000 gastroenterology outpatients. Lancet 1983;1:632-634.

7. Ferguson A, Sircus W, Eastwood MA. Frequency of "functional" gastrointestinal disorders. Lancet 1977;2:613-614.

8. Fielding JF. A year in out-patients with the irritable bowel syndrome. Ir J Med Sci 1977;146:162-166.
9. Quigley EM. Changing face of irritable bowel syndrome. World J Gastroenterol 2006;12:15.

10. Tanaka Y, Kanazawa M, Fukudo S, Drossman DA. Biopsychosocial model of irritable bowel syndrome. J Neurogastroenterol Motil 2011;17: 131-139.

11. Sykes MA, Blanchard EB, Lackner J, Keefer L, Krasner S. Psychopathology in irritable bowel syndrome: support for a psycho physiological model. J Behav Med 2003;26:361-372.

12. Drossman DA, Toner BB, Whitehead WE, et al. Cognitive-behavioral therapy versus education and desipramine versus placebo for moderate to severe functional bowel disorders. Gastroenterology 2003;125:19-31.

13. Lydiard RB, Falsetti SA. Experience with anxiety and depression treatment studies: implications for designing irritable bowel syndrome clinical trials. Am J Med 1999;107(5A):65S-73S.

14. Jafari P, Asadollahi Z, Moini M, Seyed Mirzaie M. Health related quality of life in Iranian patients with irritable bowel syndrome: reliability and validity of the persian version of the IBS-QOL. Iran Red Crescent Med J 2013;15:723-728.

15. Heaton KW, O’Donnell LJ, Braddon FE, Mountford RA, Hughes AO, Cripps PJ. Symptoms of irritable bowel syndrome in a British urban community: consulters and nonconsulters. Gastroenterology 1992;102: 1962-1967.

16. Masand PS, Kaplan DS, Gupta S, et al. Major depression and irritable bowel syndrome: is there a relationship? J Clin Psychiatry 1995;56:363367.

17. Kuppuswamy B. Manual of Socioeconomic Status (urban). Delhi: Manasayan 1981:66-72.

18. Mishra D, Singh HP. Kuppuswamy's socioeconomic status scale - A revision. Indian J Pediatr 2003;70:273-274.

19. Drossman DA, ROME III: The functional gastrointestinal disorders. McLean, VA: Degnon Associates 2006.

20. Amorim P. Mini International Neuropsychiatric Interview (MINI): validation of a short structured diagnostic psychiatric interview. Revista Brasileira de Psiquiatria 2000;22:106-115.

21. Sheehan DV, Lecrubier Y, Sheehan KH, et al. The Mini International Neuropsychiatric Interview (M.I.N.I): the development and validation of structured diagnostic psychiatric interview for DSM-IV and ICD-10. J Clin Psychiatry 1998;59(suppl 20):22-33.

22. Fukudo S, Kaneko H, Akiho H, et al. Evidence-based clinical practice guidelines for irritable bowel syndrome. J Gastroenterol 2015;50:11-30

23. Spiller R, Aziz Q, Creed F, et al. Guidelines on the irritable bowel syndrome: mechanisms and practical management. Gut 2007;56:17701798.

24. Lovell RM, Ford AC. Effect of gender on prevalence of irritable bowel syndrome in the community: systematic review and meta-analysis. Am J Gastroenterol 2012;107:991-1000.

25. Kibune Nagasako C, Garcia Montes C, Silva Lorena SL, Mesquita MA. Irritable bowel syndrome subtypes: clinical and psychological features, body mass index and comorbidities. Rev Esp Enferm Dig 2016;108:59-64

26. Earls F. Sex differences in psychiatric disorders: origins and developmental influences.Psychiatr Dev 1987;5:1-23. 
27. Mulak A, Taché Y, Larauche M. Sex hormones in the modulation of irritable bowel syndrome. World J Gastroenterol 2014;20:2433-2448.

28. Haug TT, Mykletun A, Dahl AA. The association between anxiety, depression, and somatic symptoms in a large population: the HUNT-II study. Psychosom Med 2004;66:845-851.

29. Saito YA, Schoenfeld P, Locke GR 3rd. The epidemiology of irritable bowel syndrome in North America: a systematic review. Am J Gastroenterol 2002;97:1910-1915.

30. Lin S, Mooney PD, Kurien M, Aziz I, Leeds JS, Sanders DS. Prevalence, investigational pathways and diagnostic outcomes in differing irritable bowel syndrome subtypes. Eur J Gastroenterol Hepatol 2014;26:1176-1180.

31. Su AM, Shih W, Presson AP, Chang L. Characterization of symptoms in irritable bowel syndrome with mixed bowel habit pattern. Neurogastroenterol Motil 2014;26:36-45.

32. Keshteli AH, Dehestani B, Daghaghzadeh H, Adibi P. Epidemiological features of irritable bowel syndrome and its subtypes among Iranian adults. Ann Gastroenterol 2015;28:253-258.

33. Yao X, Yang YS, Cui LH et al. Subtypes of irritable bowel syndrome on Rome III criteria: a multicenter study. J Gastroenterol Hepatol 2012;27:760-765.

34. Hausteiner-Wiehle C, Henningsen P. Irritable bowel syndrome: relations with functional, mental, and somatoform disorders. World J Gastroenterol 2014;20:6024-6030.

35. Toner BB, Garfinkel PE, Jeejeebhoy KN. Psychological factors in irritable bowel syndrome. Can J Psychiatry 1990;35:158-161.

36. Singh P, Agnihotri A, Pathak MK, et al. Psychiatric, somatic and other functional gastrointestinal disorders in patients with irritable bowel syn- drome at a tertiary care center. J Neurogastroenterol Motil 2012;18:324331.

37. Amin S, Khan AW. Life in conflict: characteristics of depression in Kashmir. Int J Health Sci (Qassim) 2009;3:213-223.

38. Margoob MA, Shiekh AA. Community prevalence of adult PTSD in south Asia- experience from Kashmir. JK Practitioner 2006;13(suppl 1):S25-S28.

39. Barsky AJ. Assessing the new DSM-5 diagnosis of somatic symptom disorder. Psychosom Med 2016;78:2-4.

40. World Health Organization. Gender and mental health. In: Wilkinson R, Marmot M, eds. Social determinants of health: the solid facts. 2nd ed. Geneva: World Health Organization 2003. Available from URL:Http:// www.who.int/gender/other_health/en/genderMH.pdf (accessed 18 Jul 2017).

41. Mayer EA, Craske M, Naliboff BD. Depression, anxiety and the gastrointestinal system. J Clin Psychiatry 2001;62(suppl 8):28-36; discussion 37.

42. Lee S, Wu J, Ma YL, Tsang A, Guo WJ, Sung J. Irritable bowel syndrome is strongly associated with generalized anxiety disorder: a community study. Aliment Pharmacol Ther 2009;30:643-651.

43. Kabra N, Nadkarni A. Prevalence of depression and anxiety in irritable bowel syndrome: a clinical based study from India. Indian J Psychiatry 2013;55:77-80.

44. Kibune Nagasako C, Garcia Montes C, Silva Lorena SL, Mesquita MA. Irritable bowel syndrome subtypes: clinical and psychological features, body mass index and comorbidities. Rev Esp Enferm Dig 2016;108:59-64. 